\title{
CONE BEAM COMPUTED TOMOGRAPHIC ASSESSMENT OF THE ANATOMICAL CHARACTERISTICS OF THE LINGUAL FORAMEN AND ITS BONY CANAL IN THE MEDIAN REGION OF THE MANDIBLE IN A GROUP OF EGYPTIAN PATIENTS
}

\author{
Sahar H. El Dessouky *
}

\begin{abstract}
The mandibular interforaminal region has long been considered as the safest area for bone harvesting and implant placement. However, this region includes some important structures such as the lingual foramen and canal. With the increasing use of implants, the number of reported postoperative complications has been rising, therefore, preoperative assessment is a critical component of dental implant surgery to prevent the possibility of neurovascular accidents and complications.
\end{abstract}

Objectives: This study was undertaken to investigate the frequency and assess the anatomical characteristics of the lingual foramina and their associated bony canals in a group of Egyptian patients using $\mathrm{CBCT}$ and to determine the relation of age and gender to these characteristics.

Material and Methods: CBCT scans of 44 Egyptian males and 56 Egyptian females were examined to assess the frequency, distribution, diameter and vertical position of the lingual foramen as well as the course, length, angulation, bifurcation of the canal and the amount of bone buccal to it.

Results: of the 100 examined cases, $44.0 \%$ showed 1 lingual foramen, $44.0 \%$ showed 2 lingual foramina, $9.0 \%$ showed 3 lingual foramina and 3.0\% showed 4 lingual foramina. $93.0 \%$ were superior and $49.0 \%$ were inferior. The mean distance to the alveolar crest was $18.2 \pm 4.1 \mathrm{~mm}$ and to the menton was $13.1 \pm 3.0 \mathrm{~mm}$ and the mean canal length was $7.3 \pm 2.4 \mathrm{~mm} .90 \%$ of the canals ran downwards, $5.0 \%$ upwards, $5.0 \%$ horizontal and in the axial plane $16.0 \%$ of the canals ran to the right, $17.0 \%$ to the left while $67.0 \%$ ran anteriorly and only $21.0 \%$ presented bifurcations. The mean vertical angulation was $60.0 \pm 20.4$ degrees and the mean horizontal angulation was $4.1 \pm$ 6.9 degrees. The mean canal diameter was $0.94 \pm 0.27 \mathrm{~mm}$ and the mean distance buccal to the foramen was $6.3 \pm 1.8 \mathrm{~mm}$. There was a statistically significant negative correlation between age and superior distance and a statistically significant positive correlation between age and inferior distance while no correlation was found between gender and any characteristic.

Conclusions: The lingual foramina and canals present many anatomical characteristics and variations and the CBCT images were able to demonstrate them. The clinical use of CBCT to evaluate each case individually is of utmost importance during preoperative planning to avoid postoperative complications bringing the patients' care to a higher standard of safety and perfection.

* Associate Professor Department of Oral Radiology, Faculty of Oral and Dental Medicine, Cairo University 


\section{INTRODUCTION}

Nowadays, dental implants are regarded as the standard option for the prosthetic rehabilitation of edentulous patients. In most cases, implant placement is a routine and predictable technique ${ }^{(1)}$. Nevertheless, preoperative assessment is a critical component of dental implant surgery to prevent the possibility of neurovascular accidents and complications $^{(2)}$. This explains the increased interest in studying the anatomy of the orofacial region since defining the anatomy can help the clinician take the correct decisions ${ }^{(3,4)}$.

The mandibular interforaminal region has long been considered as the safest area for bone harvesting and implant placement, with implant length extending up to the inferior border of mandible, while involving few risks of damage to vital anatomic structures during the surgical procedure ${ }^{(5-9)}$. However, these safety recommendations are not based on profound knowledge of the position and course of the anatomical landmarks and this region includes some important structures, such as the incive canal, the concavity of the lingual cortex and the lingual foramina and canals ${ }^{(4,10,11)}$.

The lingual foramen is a significant anatomic structure present along the midline of the lingual aspect of the mandible. The foramen, and the related vessels and nerves, are not often mentioned in descriptions of the mandibular surgical procedures, not being considered important from a clinical perspective, and even dental anatomy textbooks sometimes fail to report on their existence. However, the lingual foramen is well identified on oral radiographs and thus clearly described in textbooks related to the radiographic anatomy ${ }^{(3,5,12)}$.

This foramen penetrates the cortex on the lingual side of mandible, in the incisors' region, near the mental spines. The first author who made a deep illustration of this anatomical structure

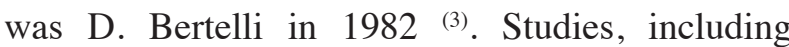
both anatomical dissection of the lower jaw and radiological evaluation with $\mathrm{CT}$, have defined and catalogued the presence of these foramina and they established a classification as well: the foramina located above the genial spines are named superior genial spinal, superior retromental, or supraspinous foramina, while the ones located below the genial spines are named inferior genial spinal, inferior retromental or infraspinous foramina ${ }^{(3,13)}$.

The content of this foramen has been a matter of debate. Some studies assume it is a vascular content, being an anastomosis of the sublingual branches of the right and left lingual arteries ${ }^{(14-16)}$. Others suggest that the superior foramina involve branches of lingual artery and vein, while the inferior ones involve branches of sublingual artery and vein and sometimes the submental vessels and branches of the mylohyoid nerve ${ }^{(6,17-19)}$. Some cadaver studies have indicated that these foramina are penetrated by branches from the sublingual artery (branch of the lingual artery) or submental artery (branch of the facial artery) or branches resulting from the anastomosis between these vessels ${ }^{(5)}$. The structures associated with the foramen were sometimes described as a neurovascular bundle ${ }^{(20)}$, which was confirmed by studies of the histological findings ${ }^{(19)}$ and by high-resolution magnetic resonance imaging ${ }^{(21)}$.

The distribution of the vessels is very complex and varies markedly in this region ${ }^{(3)}$, but those lingual vascular canals in the middle of the mandible are probably responsible for the arterial blood supply of this region and their anatomy has definite surgical implications in implant therapy and bone grafting techniques ${ }^{(3,20)}$.

With the increasing use of implants and grafting procedures for anterior jaw bone, the number of reported postoperative complications has been rising ${ }^{(21)}$. The reported neurosensory disturbances include alteration or loss of pulp sensitivity in the lower front teeth ${ }^{(22)}$.The involved artery could be of sufficient size to provoke hemorrhage intraosseously or in the connective soft tissue of 
the floor of the mouth, which might be difficult to control ${ }^{(9,23)}$ and, in some situations, even with a minimal perforation of the lingual cortical plate, life threatening hemorrhagic episodes may occur while placing the dental implants and hematoma may develop with progressive swelling of the floor of the mouth that may cause upper airway

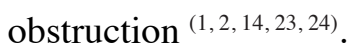

It is therefore of utmost importance for the dentist to be aware of the presence of these anatomical structures in the anterior mandible and to carefully examine the region when considering implant therapy or bone grafting techniques. Radiological imaging is highly recommended to assess the existence, number and size of the lingual foramina ${ }^{(4,8,25)}$.

Many imaging modalities have been reported to be useful for preoperative dental implant evaluation ${ }^{(10,26)}$. Periapical radiography is considered to be the first screening technique designed to display individual teeth and their supporting structures while offering unique advantages including highresolution and sharpness of the images. However, even with the long cone technique, slight image distortion can occur due to faulty angulations and this may account for the inability to precisely detect the anatomical landmarks and the limited accuracy in their measurements ${ }^{(27)}$.

Panoramic radiographs are routinely used to locate anatomical landmarks for planning surgical procedures and placement of implants in the jawbones ${ }^{(9,28)}$. However, like any other imaging modality, they have their own limitations. Reduced image sharpness caused by the use of intensifying screens, lower resolution, higher distortion and potential of overlapping anatomical structures are among those limitations (28). They can offer little information on the presence of the lingual vascular canals, due to the superimposition of the cervical vertebrae and, if the X-ray beam is not parallel to the canal, the canals cannot be depicted ${ }^{(9)}$. Besides, differential vertical and horizontal magnification factors, and operator error in patient positioning affect the utility of panoramic images to provide accurate measurements ${ }^{(29)}$.

Regardless of the technique, plain radiography has only a limited capability in the evaluation of 3D relationships needed for optimal preoperative planning of dental implant placement. MSCT and, more recently, CBCT examination have been successfully used for this purpose ${ }^{(10,27)}$.

The use of computed tomography (CT) revolutionized the dentists' ability to virtually dissect the maxillofacial structures and to determine osseous architecture without distortion. It also provides highly accurate measurements with no significant difference from the actual landmarks ${ }^{(26)}$. However, CT imaging has some major drawbacks namely the relatively high radiation dose, the high degree of background scatter around metallic restorations, the high examination costs, and the large equipment size ${ }^{(25,30)}$.

The introduction of cone beam computed tomography (CBCT) created an innovation in maxillofacial imaging and its increased usage has led to various studies of the medial region of the mandible ${ }^{(4,25,27)}$.

CBCT is an image scanning and volumetric reconstruction technique that uses a cone-shaped $\mathrm{X}$-ray beam and a scan range with a small field of view (FOV) that can be selected according to the size of the area of interest in a scanning time comparable to panoramic radiography ${ }^{(27,31,32)}$. This FOV restriction, together with the rapid image acquisition, result in a much lower radiation dose than $\mathrm{CT}^{(26,27,31)}$. The reported reduction ranges from two to three times, to as much as six times, without loss of the diagnostic information ${ }^{(27)}$. Some researchers would go to a significant reduction by up to $98 \%$ compared to the "conventional" fan-beam CT systems ${ }^{(30,33)}$, thus minimizing the effective patient dose to approximately that of a film-based 
periapical survey of the dentition ${ }^{(33)}$ or 4-15 times that of a single panoramic radiograph ${ }^{(34)}$.

All CBCT units provide voxel resolutions that are isotropic surpassing the anisotropic voxels of CT. This produces sub-millimeter spatial resolution ranging from $0.4 \mathrm{~mm}$ to as low as $0.125 \mathrm{~mm}^{(30}$, 31) thus yielding radiographic images with a high degree of resolution which enable the clinicians to identify craniofacial landmarks and allow them to obtain spatially accurate linear measurements in three dimensions ${ }^{(26,35-37)}$. Consequently, the surgeon would have the ability to accurately preplan for implant placement and even to place implants in a virtual model in terms of bone height, bone width, nerve position, and even take objective measures of bone quality ${ }^{(38)}$.

The technique allows multiplanar reformation and images are created, not only in the axial plane, but also in the coronal, sagittal and even oblique or curved image planes as well as in 3-D reconstructions ${ }^{(30,31,39)}$. The benefits of CBCT also include lower cost, smaller device size properly suited for dental clinics and a software providing some useful tools for clinical practice, such as tools to measure distances and angles, to zoom, invert the gray scale, adjust the contrast, and gamma changes ${ }^{(30,40)}$.

Hence, this study was undertaken to investigate the frequency and assess the anatomical characteristics of the lingual foramen and its associated bony canal in a group of Egyptian patients using CBCT and to determine the relation of age and gender to these characteristics.

\section{MATERIAL AND METHODS}

CBCT scans of Egyptian 44 males and 56 females (ages ranging from 15 to 77 years) were included in this study. All patients were submitted for CBCT scanning for diagnostic purposes other than the current study. All CBCT images were acquired using a Next Generation i-CAT scanner (Imaging
Sciences International, Inc., Hatfield, USA). A scout view was obtained and adjustments were made to ensure that all the patients were correctly aligned in the scanner according to the adjustment light beam before acquisition. The machine is supplied with Amorphous Silicon Flat Panel Sensor with Cesium Iodide (CsI) scintillator, $0.5 \mathrm{~mm}$ focal spot size, 14 Bit gray scale resolution, and was operated at the following protocol for all the scans of the study:

\begin{tabular}{|l|l|}
\hline Tube voltage & $120 \mathrm{kVp}$ \\
\hline Milliampere & $37.07 \mathrm{mAs}$ \\
\hline Voxel size & $0.25 \mathrm{~mm}$ \\
\hline Scanning time & 26.9 seconds \\
\hline Field of view & $10 \mathrm{~cm}$ Height $* 16 \mathrm{~cm}$ Diameter \\
\hline
\end{tabular}

After acquisition, the data were exported and transferred in DICOM format and downloaded via a Compact Disk (CD) to a personal computer for linear and angular measurements, where, Invivo Dental software (version 5.1; Anatomage, San Jose, CA, USA) was utilized.

At the software interface, the data were manipulated at the section module, where all the captured data were presented at the axial, coronal and sagittal views. The volume was then oriented to accommodate the configuration of the lingual foramen at the three views by the reorientation tool on the software task panel. But this orientation was made for each measurement solely as certain landmarks were considered in the orientation according to each measurement individually taken as will be clarified later. At each view, certain measurements were taken.

\section{Assessment on the sagittal view}

On the sagittal view, the relation of the lingual foramen to the genial tubercle (superior andlor inferior), its number, diameter and the course of 
the canal (ascending, descending or horizontal) were noted. Also the canal length, the bone buccal to it, its vertical angle and the vertical position of the foramen (distance superior and inferior to it) were measured on the same view.

\section{Relation to the genial tubercle:}

At the axial view, the sagittal reference line was made tangential to the lingual canal, then on the produced sagittal view, the relation of the foramen to the genial tubercle (superior andlor inferior) was assessed by visualizing the relation between the two landmarks and recording the finding and scrolling right and left to different levels to ensure full inspection of the area of interest. The same screening was made at the axial and coronal views to detect the presence and position of accessory canals (right andlor left). The total number of the canals was noted (Figure 1).

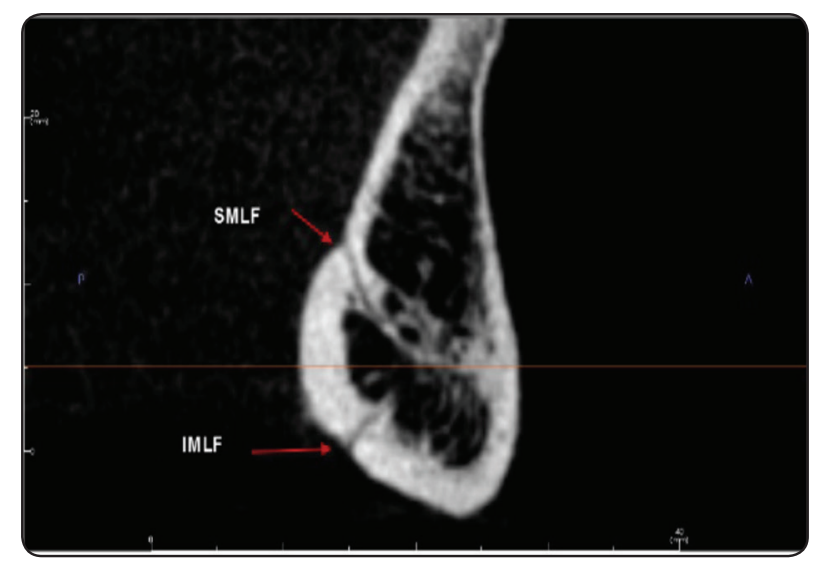

Fig. (1) Sagittal view revealing two lingual canals, one superior and one inferior to the genial tubercle. The superior one has a descending course while the inferior one is ascending.

The canal chosen for further evaluation was the superior one in the midline. If not present, then the inferior one in the midline was assessed.

\section{The course}

The course of the canal was determined, whether ascending, descending or horizontal (Figure 1).

\section{The diameter}

The number of sagittal cuts showing the foramen was noted and on the middle one the diameter of the foramen was measured as the actual distance from its upper border to its lower border (Figure 2).

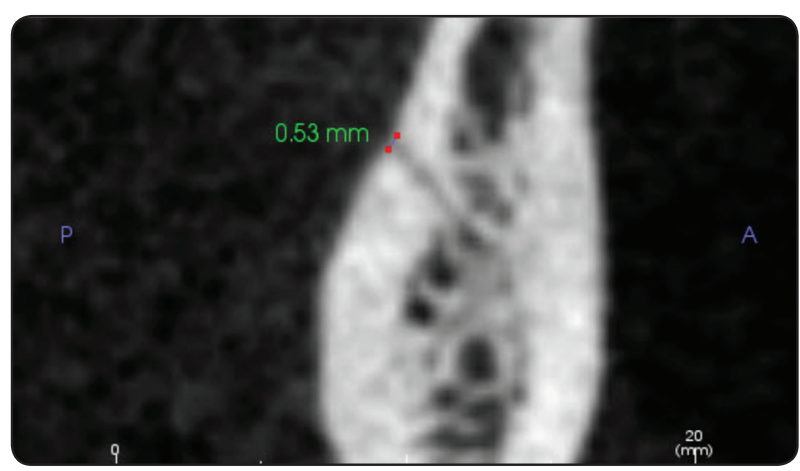

Fig. (2): Sagittal view showing measurement of the foramen diameter.

\section{The length of the canal}

The length of the canal was measured as the actual distance between the lingual foramen and its end as visualized (Figure 3).

\section{The amount of buccal bone}

The amount of bone buccal to the canal end was measured as the horizontal distance between two tangential parallel lines, one of them passing by the buccal end of the canal and the other line touching the buccal plate of bone at the pogonion level (Figure 3).

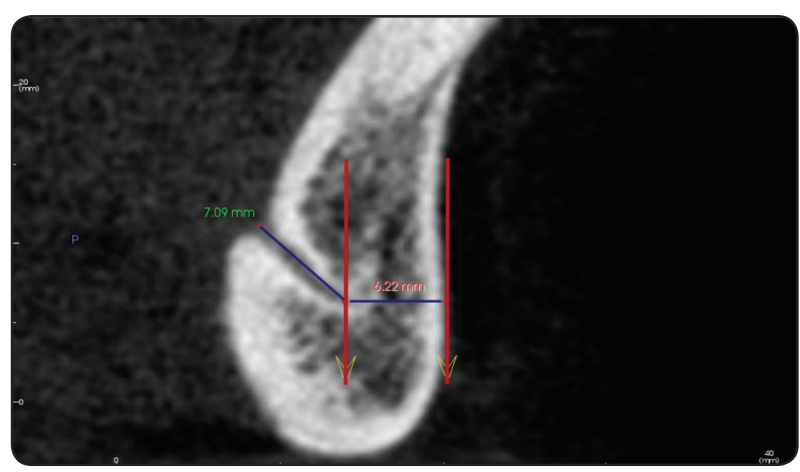

Fig. (3): Sagittal view showing the measurement of the canal length and the amount of bone buccal to it. 


\section{The vertical position of the foramen:}

For measuring the vertical position of the foramen, the volume was again reoriented in such a way as to make the coronal line passing through the crest of the ridge at the midline and the menton. At the produced sagittal view, two parallel reference lines were drawn: one representing the crest of the ridge, and the other representing the menton. At the level of the lingual foramen, a third line was drawn parallel to both reference lines. The vertical distance was measured between these three lines as the distance superior and inferior to the lingual foramen (Figure 4).

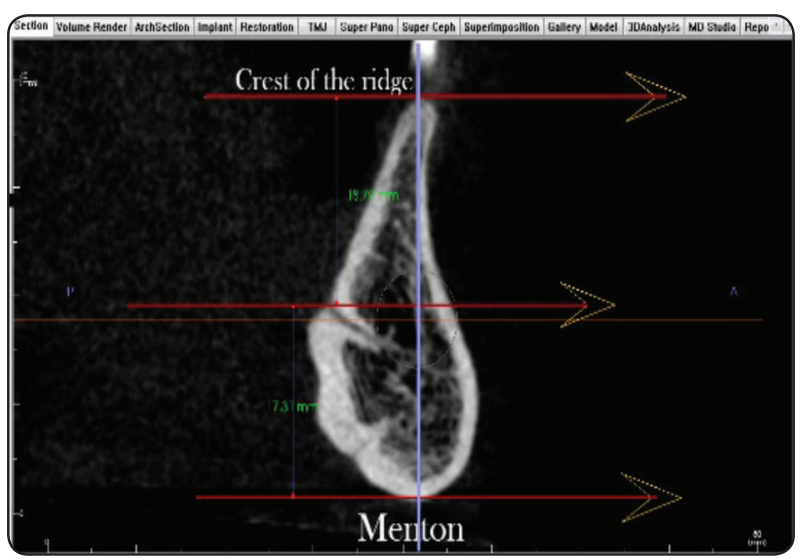

Fig. (4): Sagittal view showing the coronal line passing vertically from the crest of the ridge till the menton, and the measurement of the vertical position of the foramen.

\section{The vertical angulation of the canal}

Using the same two reference lines, a third line was drawn extending between them and perpendicular to both. The angle formed between this line and a line passing through the whole length of the canal was measured (Figure 5).

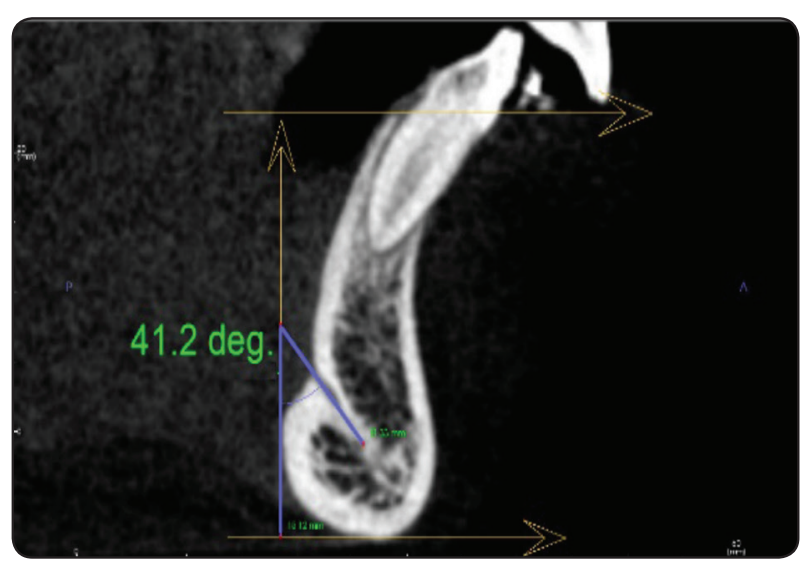

Fig. (5): Sagittal view showing the measurement of the vertical angulation of the canal.

\section{Assessment on the axial view}

On the axial view, the presence of accessory foramina, their number and relation to the genial tubercle (right and lor left) were noted while scrolling up and down to different levels to ensure full inspection of the area of interest. As well, the course of the canal (right, left or straight) and its horizontal angulation were evaluated.

\section{The horizontal angle}

First the axial plane was adjusted to view the full length of the canal. The midline was then determined by a line (sagittal reference line) passing through the pogonion and the genial tubercle. When these two landmarks were not on the same level as the lingual canal, the level was moved to them and the orientation adjusted, then the level was brought back again to the lingual canal. The angle between that line and the course of the canal was assigned as the horizontal angle of the lingual canal and its course was evaluated whether straight, to the right or the left of the reference line. (Figure $6 \mathrm{a}, \mathrm{b}$ ). 


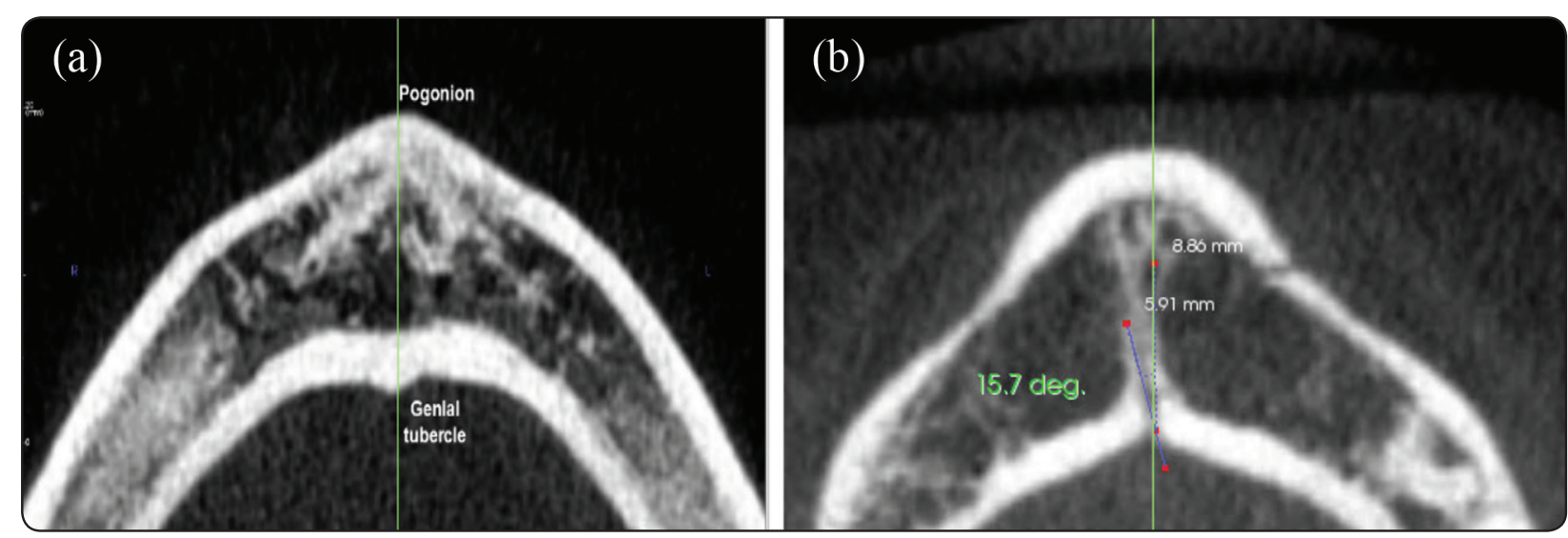

Fig. (6): Axial views showing (a) the reference line passing through the pogonion and the genial tubercle, representing the midline, and (b) measurement of the horizontal angulation of a canal that is directed to the left.

\section{The bifurcation}

The incidence of bifurcation was screened and detected whenever found on both coronal and sagittal views according to its anatomical configuration.

\section{Statistical analysis}

Numerical data were presented as mean, standard deviation (SD) and range values. Data were explored for normality by checking the data distribution and using Kolmogorov-Smirnov and Shapiro-Wilk tests. Spearman's correlation coefficient was used to determine correlations between age and different measurements. Student's t-test (for parametric data) and Mann-Whitney U test (for non-parametric data) were used for comparisons between different measurements in males and females.

The significance level was set at $\mathrm{P} \leq 0.05$. Statistical analysis was performed with $\mathrm{IBM}^{\circledR}$ SPSS ${ }^{\circledast}$ Statistics Version 20 for Windows.

\section{RESULTS}

The present study was conducted on 100 subjects; 44 males (44.0\%) and 56 females (56.0\%). The mean and standard deviation values of age were
$42.9 \pm 16.5$ years with a minimum of 15 years and a maximum of 77 years old.

As regards the number of lingual foramina; 44 subjects (44.0\%) showed 1 lingual foramen, 44 subjects (44.0\%) showed 2 lingual foramina, 9 subjects $(9.0 \%)$ showed 3 lingual foramina while only 3 subjects (3.0\%) showed 4 lingual foramina.

The superior and inferior lingual foramina prevalence in the present study were $93.0 \%$ and $49.0 \%$, respectively.

Accessory foramina were observed on the left in $13.0 \%$ of the subjects and on the right in $16.0 \%$ of the subjects.

The mean and standard deviation values of superior distance were $18.2 \pm 4.1 \mathrm{~mm}$ with a minimum of $6.2 \mathrm{~mm}$ and a maximum of $29.5 \mathrm{~mm}$. The mean and standard deviation values of inferior distance were $13.1 \pm 3.0 \mathrm{~mm}$ with a minimum of 3.0 $\mathrm{mm}$ and a maximum of $18.3 \mathrm{~mm}$.

The mean and standard deviation values of canal length were $7.3 \pm 2.4 \mathrm{~mm}$ with a minimum of 2.5 $\mathrm{mm}$ and a maximum of $13.3 \mathrm{~mm}$. 
Ninety percent of the canals had course running downwards, $5.0 \%$ were directed upwards while $5.0 \%$ were horizontal.

As regards the canal trajectory in the axial plane; $16.0 \%$ of the canals had a trajectory to the right, $17.0 \%$ were to the left while $67.0 \%$ were oriented anteriorly.

Seventy-nine percent of the canals had a single trajectory, while $21.0 \%$ presented bifurcations.

The mean and standard deviation values of vertical angulation were $60.0 \pm 20.4$ degrees with a minimum of 0.0 and a maximum of 132.0 degrees. The mean and standard deviation values of horizontal angulation were $4.1 \pm 6.9$ degrees with a minimum of 0.0 and a maximum of 32.0 degrees.

The mean and standard deviation values of canal diameter were $0.94 \pm 0.27 \mathrm{~mm}$ with a minimum of 0.55 and a maximum of $1.60 \mathrm{~mm}$.

The mean and standard deviation values of the distance buccal to the foramen were $6.3 \pm 1.8 \mathrm{~mm}$ with a minimum of 2.3 and a maximum of $11.0 \mathrm{~mm}$.

As regards the correlation between age and different measurements; there was no statistically significant correlation between age and different measurement except for a statistically significant negative (inverse) correlation between age and

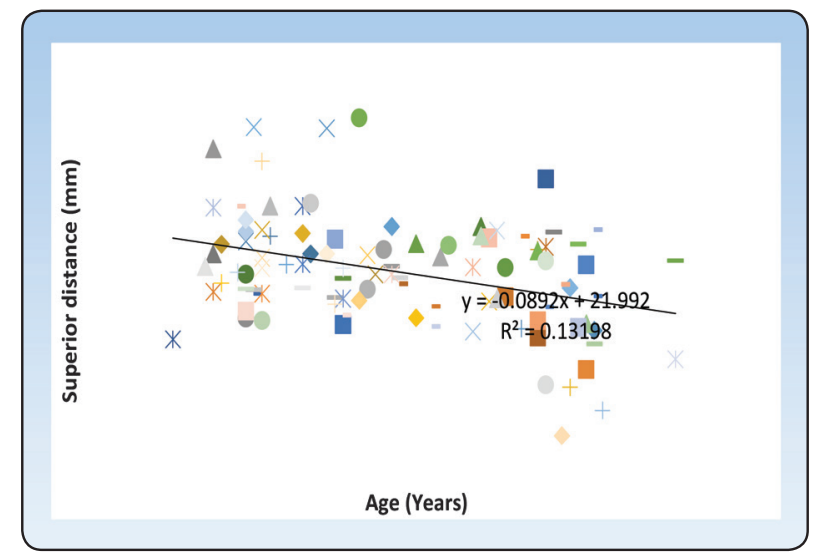

Fig. (7): Scatter diagram representing negative correlation between age and superior distance superior distance $(r=-0.308, P$-value $=0.002)$. There was also a statistically significant positive (direct) correlation between age and inferior distance $(r=0.245, P$-value $=0.014)$.

While for the gender difference; there was no statistically significant difference between males and females.

\section{DISCUSSION}

Surgical procedures in the incisors region of the mandible have long been considered safe, entailing few trivial complications. However, some studies have reported severe bleeding in this region during implant placement as a result of injury of the sublingual or submental artery resulting in lifethreatening hemorrhage in the floor of the mouth

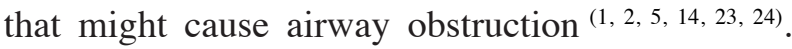
Therefore, a thorough knowledge of the anatomy of this region is highly recommended to allow for proper presurgical planning that helps the operator avoid damaging critical structures, and to enhance patients care and safety.

Panoramic radiographs were routinely used to locate the anatomical landmarks for planning surgical procedures. However, the panoramic $\mathrm{X}$-rays can offer little information on the presence of the lingual foramina and vascular canals, due to the superimposition of the cervical vertebrae and

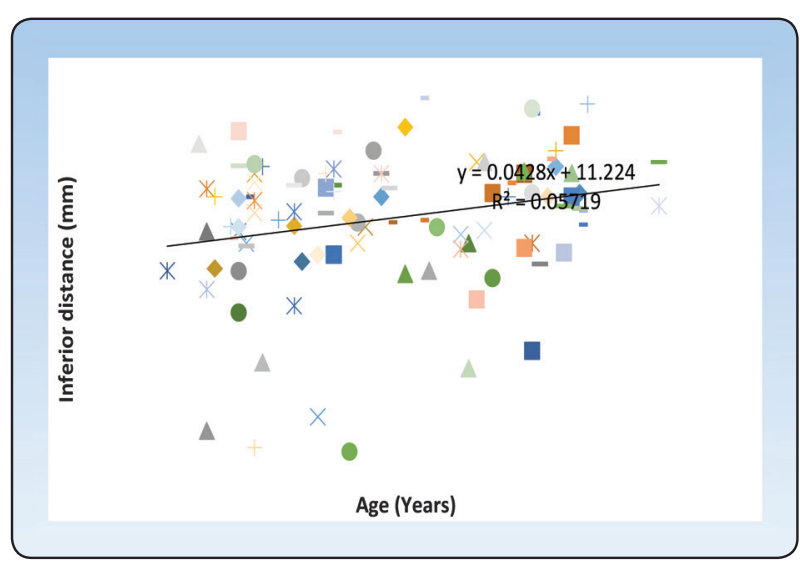

Fig. (8): Scatter diagram representing positive correlation between age and inferior distance 
to the orientation of the X-ray beam in relation to the trajectory of the canals ${ }^{(9,41)}$. Such anatomical structures are best observed with a threedimensional investigation because they dictate the appropriate size of the implant and the correct surgical procedure for the case, making it as safe as possible and reducing to a minimum the risk of serious complications ${ }^{(4)}$.

Currently CBCT is commonly used for a variety of purposes in dentomaxillofacial imaging, implantology being on top of the list ${ }^{(31,36)}$. It can reveal multiple anatomic features of the mandible that must be taken into account when planning an implant treatment such as the presence, position and size of the lingual foramen and canal in the median region of the mandible ${ }^{(4,8)}$. Moreover, a number of previous studies have proved the accuracy of linear measurements obtained by CBCT.

Of the studies conducted in this field, the one conducted by Kamburoglu, et al. ${ }^{(42)}$ can be pointed out, in which they reported that linear measurements on CBCT images yielded similar values to real ones. Also, Sumalainen A et al. ${ }^{(27)}$, Lagravère $\mathrm{MO}^{(35)}$, and Periago DR et al. ${ }^{(43)}$ concluded from their studies that the accuracy of linear measurements obtained by $\mathrm{CBCT}$ was comparable to the real values and indicated that the statistical difference between them was not clinically valuable. The same conclusion was reached by Tarazona-Álvarez $\mathrm{P}$ et al. ${ }^{(36)}$ and by Moshfeghi $\mathrm{M}$ et al.(31) who reported accuracy and reliability of CBCT linear measurements, and by Lascala $\mathrm{C}$ et al. ${ }^{(44)}$ who concluded that CBCT technique is reliable for being applied at different clinical situations where the linear measurements between anatomical sites are required since the measurements made from the CBCT images are similar, although slightly smaller, than the real ones.

Additionally, Ludlow JB et al. ${ }^{(26)}$, measured the distances between anatomic points and reference wires on dry skulls in ideal, shifted, and rotated positions using CBCT and compared them with the real measurements obtained using a digital caliper. They concluded that measurements of anatomy in CBCT volumes were relatively uninfluenced by skull orientation.

The CBCT measurements are even more accurate than those obtained by $\mathrm{CT}$ which is considered as a gold standard. This was reported by Suomalainen A et al. ${ }^{(27)}$, Kobayashi $\mathrm{k}$ et al. ${ }^{(45)}$ and Al-Ekrish A, Ekram $\mathrm{M}^{(46)}$. Moreover, many studies have also shown that linear measurement accuracy by CBCT is not influenced by metallic artifacts ${ }^{(47)}$.

In this study, CBCT examination of the median mandibular region, revealed the presence of at least one lingual foramen and canal in all the patients. This was in agreement with Sheikhi M et al. ${ }^{(8)}$ who investigated the prevalence and anatomical variations of the lingual foramen among an Iranian population and reported that, in the 102 examined cases, all the images showed at least one lingual foramen. Similarly, Babiuc I et al. ${ }^{(4)}$ reported at least one lingual foramen in $100 \%$ of their 36 cases examined by CBCT, Katakami $\mathrm{K}{ }^{(25)}$ found the lingual foramen in the midline area in all the 21 patients they examined, Tepper G et al. ${ }^{(48)}$ obtained CT images of 70 patients and observed at least one lingual foramen in $100 \%$ of the cases, Wang Y-M et al. ${ }^{(49)}$ examined the lingual foramen in a Taiwanese population using CBCT and reported that all patients exhibited at least one, and Gahleitner A et $a l .^{(50)}$, using CT, also observed at least one lingual canal in all of their 32 patients. Approaching the same results, McDonnell D et al. ${ }^{(15)}$ evaluated 314 dry skulls and reported the lingual foramina near the medial part of the mandible in 311 (99\%) of the cases, and Kawai T et al. ${ }^{(5)}$ examined 68 dry Japanese mandibles using CBCT and reported the presence of at least one foramen in $97 \%$ of the cases. However, Jacobs R et al. (51) reported that the foramen was seen in only $82 \%$ of the spiral CT images they examined. One possible explanation for this discrepancy is that the $1 \mathrm{~mm}$ slice thickness 
they used may have masked smaller diameter foramina on the mandibular midline. On the other hand, Singh N et al. ${ }^{\left({ }^{9}\right)}$ evaluated the visibility of the lingual foramen on panoramic radiographs and stated that it could not be visualized in $95.3 \%$ (286) of the cases, with poor visibility in $0.7 \%$. However, this can be easily explained on the basis of the reduced image sharpness ${ }^{(9)}$, lower resolution, higher distortion ${ }^{(28)}$ of the panoramic images as well as the superimposition of the cervical vertebrae on the median region of the mandible ${ }^{(41)}$.

In the present study, the number of foramina observed ranged from one to four with $44.0 \%$ showing 1 lingual foramen, $44.0 \%$ showing 2 lingual foramina, $9.0 \%$ showing 3 lingual foramina and only $3.0 \%$ showing 4 lingual foramina. This was in accordance with Sheikhi M et al. ${ }^{\left({ }^{(8)}\right.}$ who detected up to four lingual foramina but they found that two lingual foramina were the most frequent (52.9\%), and whenever only a single midline lingual foramen was detected, it was located superior to the genial spine. Also, Katakami K et al. ${ }^{(25)}$, in his CBCT study of the lingual foramina reported the presence of up to four foramina per patient but only in 18 of his 21 cases. This was in disagreement with Liang $\mathrm{X}$ et al. ${ }^{(52)}$ who found that a single foramen was most frequent. Babiuc I et al. ${ }^{(4)}$ reported that the number of canals found in the midline varied from one to four but with a distribution different from ours: one canal in $71.9 \%$ of the cases, two canals in $9.4 \%$, three in $15.6 \%$ and four canals in $3.1 \%$ of the cases. It is worth noting that only those patients with a single lingual foramen located inferior to the genial tubercle will enjoy the benefit of a deeper flap and a longer implant without risk of damage to the canal.

The superior and inferior lingual foramina prevalence in the present study were $93.0 \%$ and $49.0 \%$, respectively and the accessory foramina were observed on the left in $13.0 \%$ of the subjects and on the right in $16.0 \%$ of the subjects. These values were close to those of Sheikhi M et al. ${ }^{(8)}$ who re- ported a prevalence of $99 \%$ and $74.5 \%$ for the superior and inferior foramina respectively, while Kawai $\mathrm{T}$ et al. ${ }^{(5)}$, reported that the superior and inferior lingual foramen occurred in almost similar prevalence ( $86.8 \%$ and $83.8 \%$, respectively) and they observed accessory foramina on the left in $34 \%$ and on the right in $38 \%$ of the cases. Bernardi $\mathrm{S}$ et al. ${ }^{(3)}$ found that $(62 \%)$ of the examined sample had the foramen above the genial tubercle and (13\%) were below it close to the results of Babiuc I et al. ${ }^{(4)}$ who described the relation of the foramen to the genial tubercles and found it above them in $63.3 \%$ of the cases, below them in $13.34 \%$ and above and below (when there were multiple canals) in $23.3 \%$ of the cases.

In our opinion, the vertical position of the lingual foramen and canal has a greater impact in preplanning for implant placement than the number or relation to the genial tubercle as it determines the maximum length of the implant to be used while avoiding complications during surgery. In the current study, the mean distance between the lingual foramen and the alveolar crest was $18.2 \pm$ $4.1 \mathrm{~mm}$ with a minimum of $6.2 \mathrm{~mm}$ and a maximum of $29.5 \mathrm{~mm}$. In their study, Babiuc I et al. ${ }^{(4)}$ found that the mean of this distance was of a lower value, $14.2 \mathrm{~mm}$ (SD 4.34), with a minimum of 6.2 $\mathrm{mm}$ and a maximum of $26.2 \mathrm{~mm}$ while Kawai $\mathrm{T}$ et al. ${ }^{(5)}$ reported that the mean vertical distance from the superior lingual foramen to the mandibular plane was 11.43 (SD 1.46) $\mathrm{mm}$. In the current study, we found that the mean and standard deviation values of the distance between the lingual foramen and the menton were $13.1 \pm 3.0 \mathrm{~mm}$ with a minimum of $3.0 \mathrm{~mm}$ and a maximum of $18.3 \mathrm{~mm}$. Babiuc I et al. (4) found that the main foramen was at an average distance close to our results of $11.2 \mathrm{~mm}$ (SD 3.1) to the base of the mandible with a minimum of $5 \mathrm{~mm}$ and a maximum of $15 \mathrm{~mm}$, close to the values presented by Kawai T et al. ${ }^{(5)}$ who showed that the mean distance of the superior lingual foramina from the inferior mandibular plane on the Japanese dry 
mandibles was only11.43 (SD 1.56). On the other hand, Katakami K et al. ${ }^{(25)}$ found that the mean of this distance was much smaller, $7.06 \mathrm{~mm}$ with SD: 1.15. Sheikhi $\mathrm{M}$ et al. ${ }^{\left({ }^{8}\right)}$ reported that the mean distance between the superior lingual foramina from lower border of the mandible was a bit higher than ours, 14.12 (SD 2.49).

According to the results of Sheikhi M et al. ${ }^{(8)}$, the mean length of the lingual canals was 7.83 (SD $2.25) \mathrm{mm}$ which is very close to our results since we found that the mean and standard deviation values of the canal length were $7.3 \pm 2.4 \mathrm{~mm}$. However, in a macroanatomical study, Liang X et al. ${ }^{(52)}$ examined 50 dry mandibles and reported the mean length of the superior canal to be 6.8 ( $\mathrm{SD} 2.3) \mathrm{mm}$ which is much shorter.

In the current study, the mean and standard deviation values of the distance buccal to the foramen were $6.3 \pm 1.8 \mathrm{~mm}$. Babiuc I et al. ${ }^{(4)}$ stated that the lingual vascular canals traversed the bone to a variable extent where $19.4 \%$ penetrated only the lingual third of the width, $52.8 \%$ reached the middle third and $27.8 \%$ of the canals spreaded to the buccal third.

In the current study we found that the majority of the canals (90\%) were running downwards, only $5.0 \%$ were directed upwards while $5.0 \%$ were horizontal. Similarly, in their study on the Iranian population, Sheikhi M et al. ${ }^{(8)}$ reported that the majority of the superior lingual canals were running downward to the labial side and most of the inferior lingual canals were directed upward to the labial side. This was also in accordance with Kawai $\mathrm{T}$ et al. (5) who found that the canals connected to the superior lingual foramina ran downwards while those connected to the inferior foramina ran upwards. Additionally, in their macroanatomical study, Liang X et al. ${ }^{(52)}$ showed that $72 \%$ of the canals had courses running downwards to the labial side and $28 \%$ of the canals were directed upward to the labial side. On the other hand, Babiuc I et al. (4) also evaluated the orientation of the lingual vascular canal in the sagittal plane and but reported different values since only $62 \%$ of the canals had a descending trajectory, $17.3 \%$ were horizontal and $20.7 \%$ had an ascending trajectory. They also examined the canal trajectory in the axial plane, and found out that $54.5 \%$ of the canals had a trajectory slightly to the right, $36.3 \%$ were slightly to the left and $9.2 \%$ were oriented anteriorly. Once more their results were by far different from ours since we found that in the axial plane; $16.0 \%$ of the canals had a trajectory to the right, $17.0 \%$ were to the left while $67.0 \%$ were oriented anteriorly.

This justifies the fact that the lingual foramen may not be seen on periapical radiographs despite its actual presence in the patient's mandible. The genial tubercles appear as a round radiopacity in the midline of the mandible and may obscure the small radiolucency of the foramen unless the course of the canal coincides with the direction of the $\mathrm{x}$-ray beam. The vertical angulation of the Xray beam in the mandibular anterior region, using the bisecting angle technique, is negative. Consequently, the probability of projecting a clear image of the superior lingual canal is greater than the inferior one, because the Xray beam is parallel to the course of the former.

Regarding the presence of bifurcation, Babiuc I et al. ${ }^{(4)}$ reported that $87.9 \%$ of the canals had a single trajectory, while $12.1 \%$ presented bifurcations which was close to our results since we found that $79 \%$ of the canals had a single trajectory, while $21.0 \%$ presented bifurcations.

Despite the fact that the lingual foramina are frequently seen in the median region of dry mandibles or on radiological images, however, few studies have evaluated the angulation of the lingual canal $^{(7)}$. In the present study, we found that the mean and standard deviation values of vertical angulation were $60.0 \pm 20.4$ degrees which are less than the values reported by Kawai T et al. ${ }^{(5)}$ who found that 
the mean vertical angulation of the superior foramina was 77.48 (SD 12.54) degrees. Once more our values differed from theirs since they measured the horizontal angulation of the accessory canals only and found its mean to be 10.02 (SD 9.11) degrees while we measured the horizontal angulation of the main canals and found its mean $4.1 \pm 6.9$ degrees.

The diameter of these canals or foramina is related to the blood-supplying capacity of the blood vessel passing through, and as a result, if they are wide enough, they may bleed significantly if injured during the surgical procedures ${ }^{(6,23)}$. The mean and standard deviation values of canal diameter measured in this study were $0.94 \pm 0.27 \mathrm{~mm}$ with a minimum of 0.55 and a maximum of $1.60 \mathrm{~mm}$. In their study, Babiuc I et al. ${ }^{(4)}$ found that the average diameter was $0.86 \mathrm{~mm}$ (SD 0.3), ranging from 0.52 to $1.74 \mathrm{~mm}$. Bernardi $\mathrm{S}$ et al. $^{(3)}$ also measured the diameter of the lingual foramina by CBCT and reported that the mean diameter of the superior foramina was 1,24 $\pm 0,32 \mathrm{~mm}$. Additionally, Wang Y-M ${ }^{(49)}$ found that the lingual canal diameter ranged from 0.25 to $1.90 \mathrm{~mm}$ with a mean of $0.61 \mathrm{~mm}$ while the reported values of Katakami K et al. ${ }^{(25)}$ for the mean horizontal and vertical widths of the lingual foramina were $1.17 \mathrm{~mm}$ (SD: 0.28 ) and $0.88 \mathrm{~mm}$ (SD: 0.20), respectively.

We did not find any statistically significant correlation between age and the different measurements except for a statistically significant negative (inverse) correlation between age and the superior distance and a statistically significant positive (direct) correlation between age and the inferior distance. Our results were in accordance with those of Sheikhi M et al. ${ }^{(8)}$ who did not find any correlation between the age of the patients and the obtained measurements except for a weak negative correlation to the distance from the foramen to the alveolar crest indicating that, as the patient gets older, the distance of the lingual foramen to the alveolar crest decreases. The most probable explanation for this correlation is the increased alveolar bone resorption with aging.
Regarding the gender, we did not find any statistically significant difference between males and females contrary to Sheikhi M et al. ${ }^{(8)}$ who demonstrated that the distance of the buccal end of the canal to the buccal bone plate was also greater in male population than in females and also found a significant difference between gender and distance of the lingual foramen to the alveolar crest with a possible explanation that the mandible in the male Iranian population is of larger size.

\section{CONCLUSION}

In the current study, CBCT images were able to demonstrate the anatomical characteristics and variations of the lingual foramina and their associated canals and there were some agreements and some deviations from the previous studies. In our opinion, a thorough evaluation of each case individually is of utmost importance during preoperative planning, especially for implant placement to avoid postoperative complications bringing the patient's care to a higher standard of safety and perfection.

\section{REFERENCES}

1. Dubois L, De Lange J, Baas E, Van Ingen J. Excessive bleeding in the floor of the mouth after endosseus implant placement: a report of two cases. International journal of oral and maxillofacial surgery. 2010;39(4):412-5.

2. Jo J-H, Kim S-G, Oh J-S. Hemorrhage related to implant placement in the anterior mandible. Implant dentistry. 2011;20(3):e33-e7.

3. Bernardi S, Rastelli C, Leuter C, Gatto R, Continenza MA. Anterior Mandibular Lingual Foramina: An In Vivo Investigation. Anatomy research international. 2014;2014.

4. Babiuc I, Tarlungeanu I, Pauna M. Cone beam computed tomography observations of the lingual foramina and their bony canals in the median region of the mandible. Rom $\mathrm{J}$ Morphol Embryol. 2011;52(3):827-9.

5. Kawai T, Asaumi R, Sato I, Yoshida S, Yosue T. Classification of the lingual foramina and their bony canals in the median region of the mandible: cone beam computed tomography observations of dry Japanese mandibles. Oral Radiology. 2007;23(2):42-8. 
6. Angelopoulos C. Cone beam tomographic imaging anatomy of the maxillofacial region. Dental clinics of North America. 2008;52(4):731-52.

7. Liang X, Lambrichts I, Corpas L, Politis C, Vrielinck L, Ma GW, et al. Neurovascular disturbance associated with implant placement in the anterior mandible and its surgical implications: literature review including report of a case. Chinese Journal of Dental Research. 2008;11(1):56.

8. Sheikhi M, Mosavat F, Ahmadi A. Assessing the anatomical variations of lingual foramen and its bony canals with CBCT taken from 102 patients in Isfahan. Dental Research Journal. 2012;9(Suppl 1):S45.

9. Singh N, Jaju P, Jaju S, Agarwal R. Detection of anatomical variations in mandible by panoramic radiography. Journal of Cranio-Maxillary Diseases. 2014;3(2):95.

10. Naser AZ, Mehr BB. A comparative study of accuracy of linear measurements using cone beam and multi-slice computed tomographies for evaluation of mandibular canal location in dry mandibles. Dental Research Journal. 2013;10(1): 15 .

11. Pommer B, Tepper G, Gahleitner A, Zechner W, Watzek G. New safety margins for chin bone harvesting based on the course of the mandibular incisive canal in CT. Clinical oral implants research. 2008;19(12):1312-6.

12. Mraiwa N, Jacobs R, Moerman P, Lambrichts I, van Steenberghe D, Quirynen M. Presence and course of the incisive canal in the human mandibular interforaminal region: two-dimensional imaging versus anatomical observations. Surgical and Radiologic Anatomy. 2003;25(5-6):416-23.

13. Gupta S, Soni A, Singh P. Morphological study of accessory foramina in mandible and its clinical implication. Indian journal of oral sciences. 2013;4(1):12.

14. Jaju P, Jaju S. Lingual vascular canal assessment by dental computed tomography: A retrospective study. Indian Journal of Dental Research. 2011;22(2):232.

15. McDonnell D, Nouri MR, Todd M. The mandibular lingual foramen: a consistent arterial foramen in the middle of the mandible. Journal of anatomy. 1994;184(Pt 2):363.

16. Darriba MA, Mendonça-Caridad JJ. Profuse bleeding and life-threatening airway obstruction after placement of mandibular dental implants. Journal of oral and maxillofacial surgery. 1997;55(11):1328-30.

17. Yoshida S, Kawai T, Okutsu K, Yosue T, TAKAMORI H, SUNOHARA M, et al. The appearance of foramen in the internal aspect of the mental region of mandible from Japanese cadavers and dry skulls under macroscopic observation and three-dimensional CT images. Okajimas folia anatomica Japonica. 2005;82(3):83-8.

18. Kawai T, Sato I, Yosue T, Takamori H, Sunohara M. Anastomosis between the inferior alveolar artery branches and submental artery in human mandible. Surgical and Radiologic Anatomy. 2006;28(3):308-10.

19. Liang X, Jacobs R, Lambrichts I, Vandewalle G, Van Oostveldt D, Schepers E, et al. Microanatomical and histological assessment of the content of superior genial spinal foramen and its bony canal. Dentomaxillofacial Radiology. 2014 .

20. Vasconcellos HAd, Campos AEdS, Almeida GHd, Maia T, Lie M, Vasconcellos PHBd. The anatomy of the lingual foramen canal and its related to the mandibular symphysis. Rev chil anat. 2000;18(1):47-51.

21. Jacobs R, Lambrichts I, Liang X, Martens W, Mraiwa N, Adriaensens $\mathrm{P}$, et al. Neurovascularization of the anterior jaw bones revisited using high-resolution magnetic resonance imaging. Oral Surgery, Oral Medicine, Oral Pathology, Oral Radiology, and Endodontology. 2007;103(5):68393.

22. Sbordone L, Menchini-Fabris G, Toti P, Sbordone C, Califano L, Guidetti F. Clinical survey of neurosensory sideeffects of mandibular parasymphyseal bone harvesting. International journal of oral and maxillofacial surgery. 2009;38(2):139-45.

23. Kalpidis CD, Setayesh RM. Hemorrhaging associated with endosseous implant placement in the anterior mandible: a review of the literature. Journal of periodontology. 2004;75(5):631-45.

24. de Vera JDC-P, Calleja JL-A, Burgueño-García M. Hematoma of the floor of the mouth and airway obstruction during mandibular dental implant placement: a case report. Oral and maxillofacial surgery. 2008;12(4):223-6.

25. Katakami K, Mishima A, Kuribayashi A, Shimoda S, Hamada Y, Kobayashi K. Anatomical characteristics of the mandibular lingual foramina observed on limited cone-beam CT images. Clinical oral implants research. 2009;20(4):386-90.

26. Ludlow JB, Laster WS, See M, Bailey LTJ, Hershey HG. Accuracy of measurements of mandibular anatomy in cone beam computed tomography images. Oral Surgery, Oral Medicine, Oral Pathology, Oral Radiology, and Endodontology. 2007;103(4):534-42. 
27. Suomalainen A, Vehmas T, Kortesniemi M, Robinson S, Peltola J. Accuracy of linear measurements using dental cone beam and conventional multislice computed tomography. Dentomaxillofacial Radiology. 2014.

28. Iyengar AR, Patil S, Nagesh KS, Mehkri S, Manchanda A. Detection of anterior loop and other patterns of entry of mental nerve into the mental foramen: A radiographic study in panoramic images. Journal of Dental Implants. 2013;3(1):21.

29. Laster W, Ludlow J, Bailey L, Hershey HG. Accuracy of measurements of mandibular anatomy and prediction of asymmetry in panoramic radiographic images. Dentomaxillofacial radiology. 2014.

30. Scarfe WC, Farman AG, Sukovic P. Clinical applications of cone-beam computed tomography in dental practice. Journal-Canadian Dental Association. 2006;72(1):75.

31. Moshfeghi M, Tavakoli MA, Hosseini ET, Hosseini AT, Hosseini IT. Analysis of linear measurement accuracy obtained by cone beam computed tomography (CBCT-NewTom VG). Dental Research Journal. 2012;9(Suppl 1):S57.

32. Sato S, Arai Y, Shinoda K, Ito K. Clinical application of a new cone-beam computerized tomography system to assess multiple two-dimensional images for the preoperative treatment planning of maxillary implants: case reports. Quintessence international (Berlin, Germany: 1985). 2003;35(7):525-8.

33. Schulze D, Heiland M, Thurmann H, Adam G. Radiation exposure during midfacial imaging using 4-and 16-slice computed tomography, cone beam computed tomography systems and conventional radiography. Dentomaxillofacial Radiology. 2014.

34. Ngan D, Kharbanda O, Geenty J, Darendeliler M. Comparison of radiation levels from computed tomography and conventional dental radiographs. Australian orthodontic journal. 2003;19(2):67-75.

35. Lagravère MO, Carey J, Toogood RW, Major PW. Threedimensional accuracy of measurements made with software on cone-beam computed tomography images. American Journal of Orthodontics and Dentofacial Orthopedics. 2008;134(1):112-6.

36. Tarazona-Álvarez P, Romero-Millán J, Peñarrocha-Oltra D, Fuster-Torres MÁ, Tarazona B, Peñarrocha-Diago M. Comparative study of mandibular linear measurements obtained by cone beam computed tomography and digital calipers. Journal of clinical and experimental dentistry. 2014;6(3):e271.
37. Flores-Mir C, Rosenblatt MR, Major PW, Carey JP, Heo G. Measurement accuracy and reliability of tooth length on conventional and CBCT reconstructed panoramic radiographs. Dental press journal of orthodontics. 2014;19(5):45-53.

38. Quereshy FA, Savell TA, Palomo JM. Applications of cone beam computed tomography in the practice of oral and maxillofacial surgery. Journal of oral and maxillofacial surgery. 2008;66(4):791-6.

39. Cevidanes LH, Styner MA, Proffit WR. Image analysis and superimposition of 3-dimensional cone-beam computed tomography models. American journal of orthodontics and dentofacial orthopedics. 2006;129(5):611-8.

40. Brown AA, Scarfe WC, Scheetz JP, Silveira AM, Farman AG. Linear accuracy of cone beam CT derived 3D images. The Angle orthodontist. 2009;79(1):150-7.

41. Dreiseidler T, Mischkowski RA, Neugebauer J, Ritter L, Zöller JE. Comparison of cone-beam imaging with orthopantomography and computerized tomography for assessment in presurgical implant dentistry. The International journal of oral \& maxillofacial implants. 2009;24(2): 216-25.

42. Kamburoğlu K, Kılıç C, Özen T, Yüksel SP. Measurements of mandibular canal region obtained by cone-beam computed tomography: a cadaveric study. Oral Surgery, Oral Medicine, Oral Pathology, Oral Radiology, and Endodontology. 2009;107(2):e34-e42.

43. Periago DR, Scarfe WC, Moshiri M, Scheetz JP, Silveira AM, Farman AG. Linear accuracy and reliability of cone beam CT derived 3-dimensional images constructed using an orthodontic volumetric rendering program. The Angle orthodontist. 2008;78(3):387-95.

44. Lascala C, Panella J, Marques M. Analysis of the accuracy of linear measurements obtained by cone beam computed tomography (CBCT-NewTom). Dentomaxillofacial Radiology. 2014.

45. Kobayashi K, Shimoda S, Nakagawa Y, Yamamoto A. Accuracy in Measurement of Distance Using Limited ConeBeam Computerized Tomography. International Journal of Oral \& Maxillofacial Implants. 2004;19(2).

46. Al-Ekrish A, Ekram M. A comparative study of the accuracy and reliability of multidetector computed tomography and cone beam computed tomography in the assessment of dental implant site dimensions. Dentomaxillofacial Radiology. 2014. 
47. Patcas R, Müller L, Ullrich O, Peltomäki T. Accuracy of cone-beam computed tomography at different resolutions assessed on the bony covering of the mandibular anterior teeth. American Journal of Orthodontics and Dentofacial Orthopedics. 2012;141(1):41-50.

48. Tepper G, Hofschneider UB, Gahleitner A, Ulm C. Computed tomographic diagnosis and localization of bone canals in the mandibular interforaminal region for prevention of bleeding complications during implant surgery. The International journal of oral \& maxillofacial implants. 2001;16(1):68-72.

49. Wang Y-M, Ju Y-R, Pan W-L, Chan C-P. Evaluation of location and dimensions of mandibular lingual canals: a cone beam computed tomography study. International journal of oral and maxillofacial surgery. 2015.

50. Gahleitner A, Hofschneider U, Tepper G, Pretterklieber M, Schick S, Zauza K, et al. Lingual Vascular Canals of the Mandible: Evaluation with Dental CT 1. Radiology. 2001;220(1):186-9.

51. Jacobs R, Mraiwa N, VanSteenberghe D, Gijbels F, Quirynen $\mathrm{M}$. Appearance, location, course, and morphology of the mandibular incisive canal: an assessment on spiral CT scan. Dentomaxillofacial Radiology. 2002;31(5):322-7.

52. Liang X, Jacobs R, Lambrichts I, Vandewalle G. Lingual foramina on the mandibular midline revisited: a macroanatomical study. Clinical Anatomy. 2007;20(3):246-51. 